\title{
Untersuchungen zur Funktion des Mesopsammon bei der Reinigung von infiltriertem Oberflächenwasser
}

von

\author{
Barbara RITTERBUSCH*
}

\begin{abstract}
SUMMARY
The ecology of metazoa in a settling basin of the Berlin Water Works and its importance in the process of purification of percolated surface water.
\end{abstract}

Based upon the hypothesis that the metazoa (nematoda and rotifera) in the interstitial stratum of a slow sand filter are of importance in the process of purification of surface water, a filter was built to check the substrate characteristics of different grain sizes compared with the natural soil.

It was found that the granulometric composition of sands as found in the settling basins did have the best comparative screening properties. This was demonstrated by chemical analyses of $\mathrm{NH}_{4}, \mathrm{NO}_{2}, \mathrm{NO}_{3}, \mathrm{PO}_{4}, \mathrm{KMnO}_{4}$-consumption and $\mathrm{O}_{2}$-saturation. Only the $\mathrm{NO}_{3}$-graph is shown here.

The importance of metazoa in the process of filtering water was studied by inoculating metazoic microorganisms into the most efficient filter. Chemical analyses of the water did not reveal a significant change of water quality over that from a non-inoculated filter. Because of this result the metazoa do not appear to act as an important component in the water purification by sand filtration.

\section{VORBEMERKUNG}

Im vorliegenden Fall handelt es sich um das Metazoen-Mesopsammon in Sickerbecken der Berliner Wasserwerke. Ökologische Untersuchungen haben gezeigt, daß es sich mengenmäßig hauptsächlich um Nematoden, danach um Rotatorien handelt. In der Reihe folgen dann Oligochaeten und Crustaceen (Harpacticiden), allerdings in derart geringer Individuenzahl, daß ihre Wirkı:ng bei der Reinigung des infiltrierten Oberflächenwassers als vernachlässigbar klein erachtet werden kann.

Die Nematoden sind mit 10 Arten vertreten:

Monhystera subfiliformis Cobb, 1918

Theristus flevensis Schuurmans Stekhoven, 1935

* Eppingerstr. 22, 1 Berlin 33. 
Diplogaster rivalis Leydig, 1854, Butschli, 1873

Prismatolaimus intermedius Bütschli, 1873, De Man, 1880

Prismatolaimus dolichurus De Man, 1880

Stenonchulus troglodytes Schneider, 1940

Tobrilus gracilis Bastian, 1865, Andrassy, 1959

Mononchus truncatus Bastian, 1865

Mylonchulus sigmaturus Cobb, 1917, Altherr, 1953

Paractinolaimus macrolaimus De Man, 1880, Andrassy, 1964.

Hervorgehoben sei die Art Monhystera subfil., weil sie für Europa erstmalig nachgewiesen werden konnte.

Bei den Rotatorien konnten 18 verschiedene Arten nachgewiesen werden: Brachionus angularis Gosse, 1851

Br. calyciflorus Pallas, 1766

Br. urceolaris O. F. Müller, 1773

Kellicottia longispina Kellicott, 1879

Keratella cochlearis Gosse, 1851

Keratella quadrata O. F. Müller, 1786

K. ticinensis Callerio, 1920

Notommata glyphura Wulfert, 1935

Habrotrocha constricta Dujardin, 1841

Macrotrachela habita Bryce, 1894

Cephalodella gibba Ehrenberg, 1832

Encentrum grande Western, 1891

Dicranophorus forcipatus O. F. Müller, 1786

Notholca acuminata Ehrenberg, 1832

Notholca squamula O. F. Müller, 1786

Rotatoria rotatoria Pallas, 1766

Filinia terminalis Plate, 1886

Filinia longiseta Ehrenberg, 1834

\section{Bdelloidea}

Für beide Gruppen wurde festgestellt, daß zwar die mesopsammalen Arten nicht an der Oberfläche vorkommen, daß aber solche Arten, die wir als pelagisch ansehen, wie z.B. der Rotator Keratella quadrata häufig und bis in 140 $\mathrm{cm}$ Tiefe im Interstitial vorkommt. Das bedeutet, daß durch ein günstiges Verhältnis von Körpergröße der Organismen zum Porenvolumen des Substrats sowie durch gute Ernährungsbedingungen eine ständige Immigration in das Interstitial möglich ist. Feuchtsandbewohner können das Sediment bis in mehrere Meter Tiefe besiedeln. (Eigener Nachweis bis in $260 \mathrm{~cm}$ Tiefe).

Als Arbeitshypothese der nachfolgenden Untersuchungen bin ich davon ausgegangen, daß der Stoffwechsel der Metazoen besonders bei hoher Individuenzahl, von Einfluß auf die Wasserqualität ist, denn das Infiltrat (Oberflächenwasser der Faulen Spree) wird auf der Sickerstrecke soweit gereinigt, daß es nahezu Trinkwasserqualität erreicht (Grundwasser). 


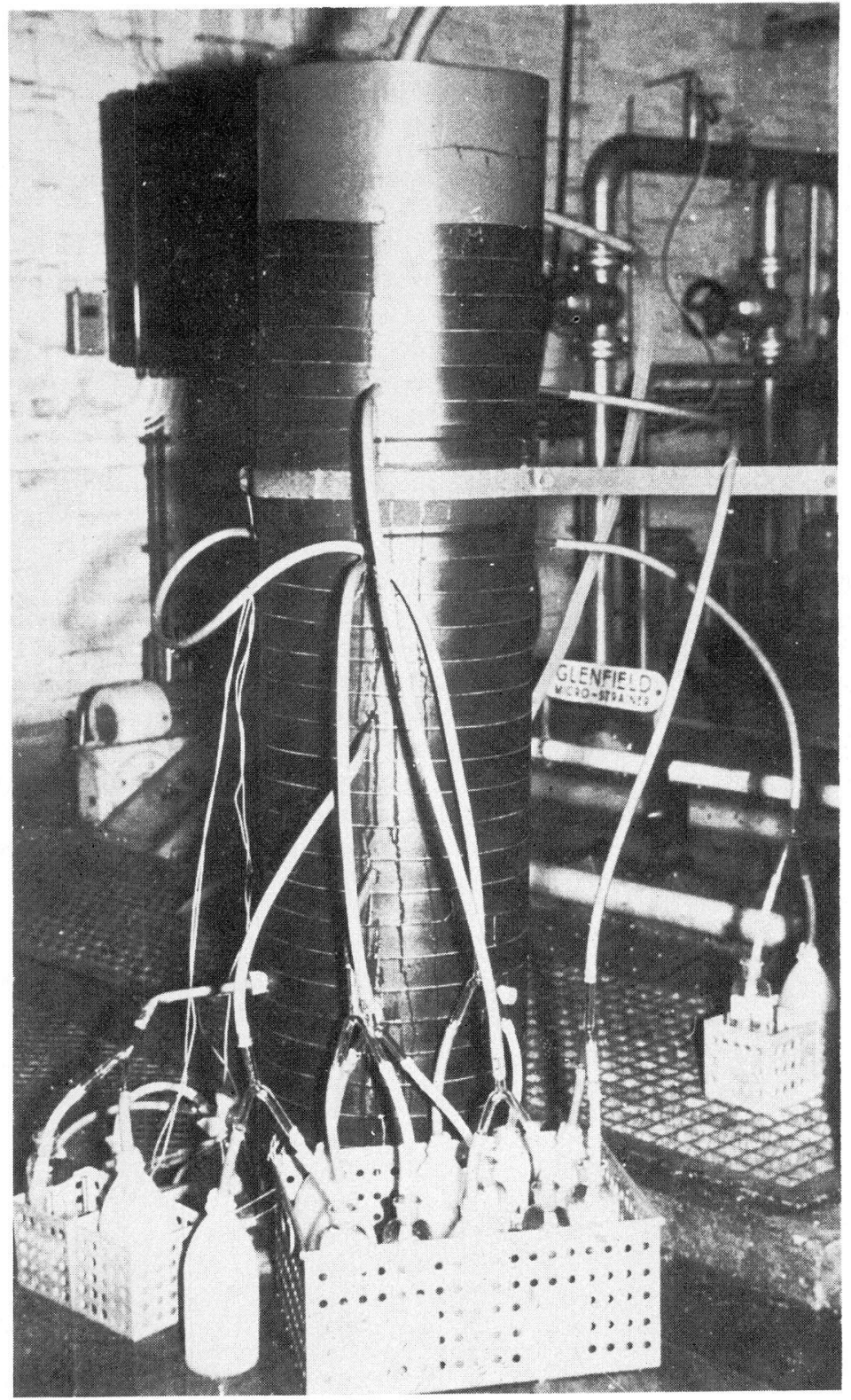

Abb. 1: Der Versuchsfilter. 
Die Untersuchungen sollten zeigen, ob die Metazoen bei der Versickerung des Wassers durch die Beseitigung von Detritus wesentlich an dessen Reinigung beteiligt sind, und ob sie möglicherweise im technischen Maßstab als biologische Wasserreiniger eingesetzt werden können.

Der erste Schritt bestand darin, ihr Biotop, d.h. die Korngrößen und entsprechend die intergranularen Hohlräume zu verändern, im wesentlichen zu vergrößern.

Als Modell habe ich einen Filter gebaut, der in verschiedenen Tiefen $(5,10$, $15,20,30,50,65,85,110 \mathrm{~cm})$ Entnahmevorrichtungen für Wasserproben hatte. (Abb. 1)

Als Maßstab für die Veränderung der Wasserqualität während der Versickerung dienten chemische Daten, die 5-7 mal während einer Laufzeit von jeweils 14 Tagen von den verschiedenen Probestellen ermittelt wurden. $\mathrm{NH}_{4}$, $\mathrm{NO}_{2}, \mathrm{NO}_{3}, \mathrm{PO}_{4}, \mathrm{KMnO}_{4}$-Verbrauch, $\mathrm{O}_{2}$-Sättigung wurden analysiert und dienten als Parameter für die Mineralisationsvorgänge im Verlauf von Zeit und Ort. In der vorliegenden Beschreibung werden lediglich die $\mathrm{NO}_{3}$-Kurven herangezogen.

Zwar muß man davon ausgehen, daß die N-Verbindungen und Phosphat im wesentlichen Produkte bakterieller Umsetzung sind und weitgehend unabhängig von der geologischen Beschaffenheit des Substrats. Da aber Teile der Metazoenfauna für die laufende Vernichtung von Detritus verantwortlich sind, bin ich davon ausgegangen, daß eine Verbesserung der Biotopeigenschaften durch Vergrößerung des Porenvolumens besonders für die größeren Organismen eine Verbesserung ihrer Lebensbedingungen bedeutet, die sich in stärkerer Freßtätigkeit ausdrückt. Das sollte sich niederschlagen in einer beschleunigten Mineralisation der organischen Reste durch die vorhandenen Bakte-

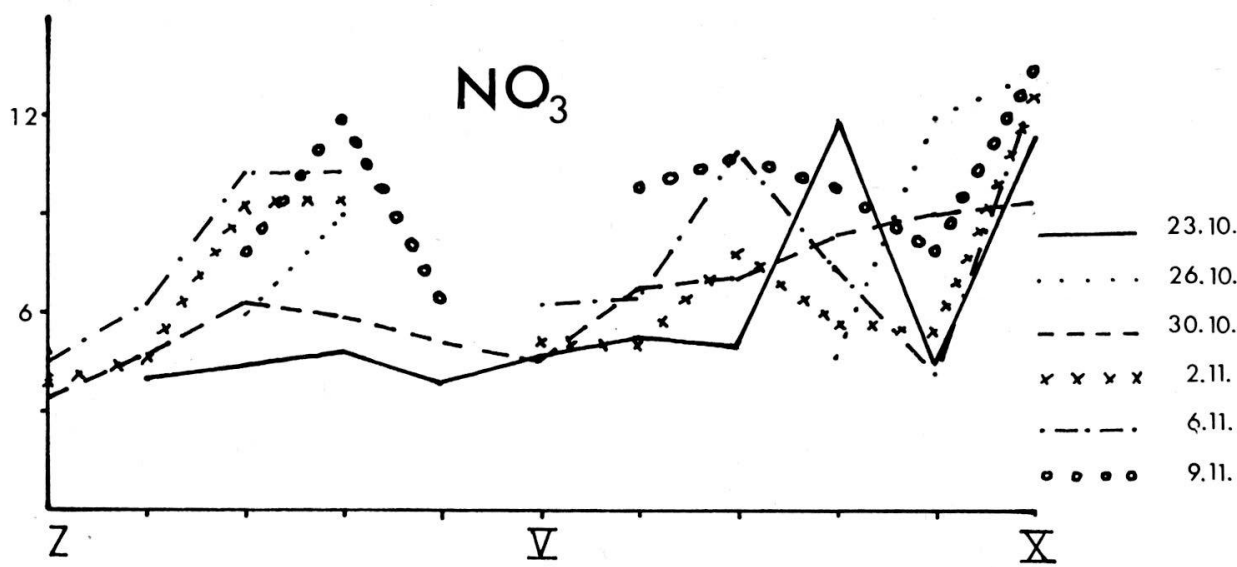

Abb. 2: Der Gehalt an $\mathrm{NO}_{3}(\mathrm{mg} / \mathrm{l})$ in den einzelnen Wasserproben zwischen Zulauf, $\mathrm{Z}$, und $\mathrm{Ab}-$ lauf, X, von Filter 1 (reiner Grobsand). 
rien. Chemisch würde sich das in einer zeitlich und örtlich schnelleren Zunahme der Oxydationsprodukte darstellen, in diesem Fall hauptsächlich die Umsetzung von $\mathrm{NH}_{4} \mathrm{zu} \mathrm{NO}$. Diese Veränderung sollte trotz Schwankungen im Detritus- und $\mathrm{NH}_{4}$-Gehalt der Vorflut im Filter deutlich erkennbar sein.

Ich habe daraufhin den ersten Filter mit reinem Grobsand (1,0-2,0 $\mathrm{mm})$ gefüllt und innerhalb von 2 Wochen 6 Wasserproben für die genannten Analysen aus allen Meßstellen analysiert. (Abb. 2)

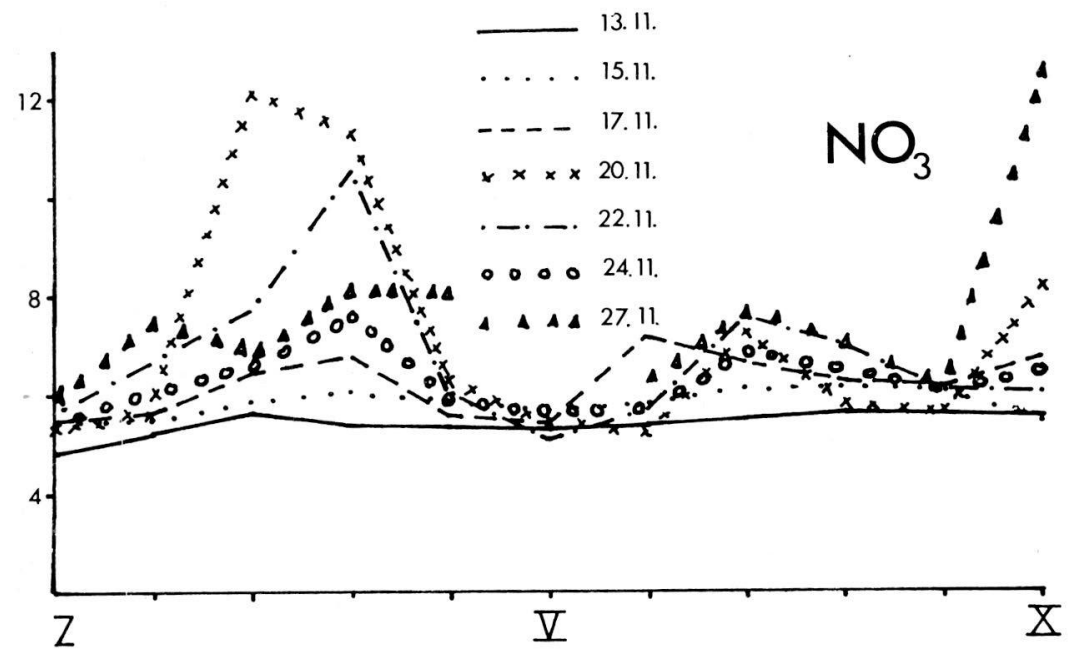

Abb. 3: Der Gehalt an $\mathrm{NO}_{3}(\mathrm{mg} / \mathrm{l})$ in den einzelnen Wasserproben zwischen Zulauf, Z, und Ablauf, X, von Filter 2 (reiner Mittelsand).

Dabei konnte nur eine sehr schwache Sieb- und Filterwirkung festgestellt werden. Eine Einarbeitung des Filters war nicht erkennbar.

Grobsand scheint also ungeeinget als Biotop für eine gut funktionierende Interstitialfauna.

Der nächste Filter wurde mit reinem Mittelsand (0,7-1,2 mm) gefüllt, entspricht als Biotop also zum großen Teil den natürlichen Sickerböden (alluviale Talsande). Dieser Filter arbeitet geringfügig besser, d.h., die Tendenz zur Mineralisation ist etwas größer als bei Filter l. (Abb. 3)

Die folgende Füllung bestand aus einer Mischung von Feinkies: Grobsand: Mittelsand: Feinsand im Verhältnis 10:40:40:10. Hierbei war festzustellen, daß sie nur wenig besser als die beiden vorherigen Filter arbeitete. (Abb. 4)

Die graphische Abbildung läßt erkennen, daß sehr viele Proben ausgefallen waren. Das lag an einer Verstopfung der Sandlücken teils durch eingetragene Luftblasen, wahrscheinlich aber auch durch Stickstoffgas, Dieses entsteht infolge einer Stickstoff-Übersättigung im Wasser, wobei der Sauerstoff durch chemische und/oder biologische Prozesse verbraucht wird und der verbleiben- 


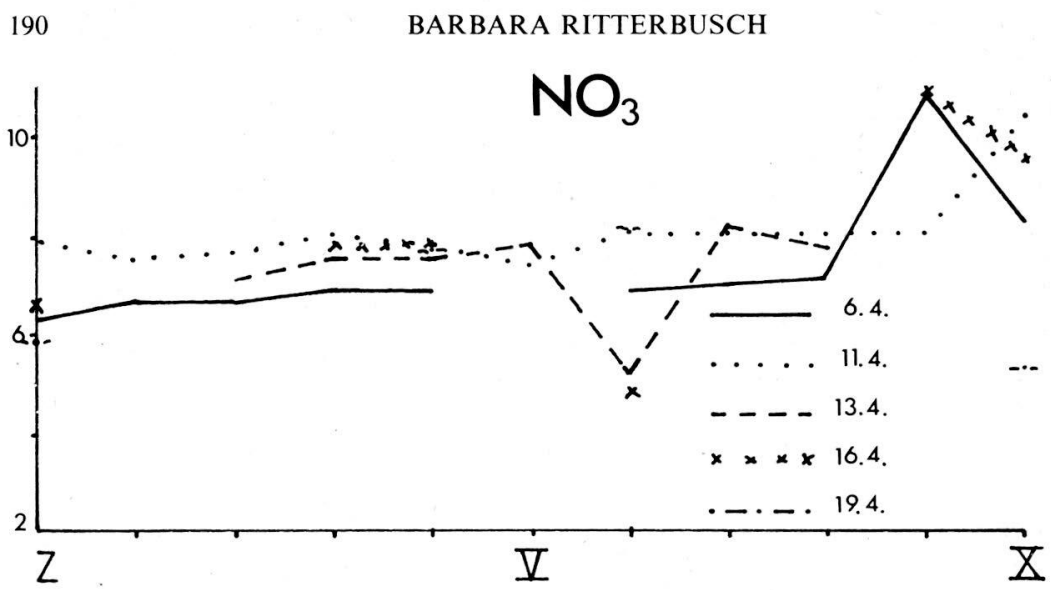

Abb. 4: Der Gehalt an $\mathrm{NO}_{3}(\mathrm{mg} / \mathrm{l})$ in den einzelnen Wasserproben zwischen Zulauf, $\mathrm{Z}$, und $\mathrm{Ab}$ lauf, X, von Filter 3 (Mischsand).

de Stickstoff sich bei Berührung mit dem Substrat abscheidet, besonders bei Temperaturerhöhung.

Dieses Problem zieht sich durch alle Filterserien und ist z.T. so schwerwiegend, daß in einigen Fällen gar keine Aussage über die Mineralisation im Filter möglich ist.

Die nächste Filterfüllung ist geschichtet: Grobsand-Mittelsand-Feinsand zu gleichen Volumina und in der Reihenfolge von oben nach unten. (Abb. 5)

Anhand der $\mathrm{NO}_{3}$-Kurve läßt sich leicht erkennen, daß kaum eine Umsetzung erfolgt ist, und daß der Filter keineswegs besser arbeitet als die vorhergehenden.

Der folgende Filter ahmt die natürlichen Verhältnisse der Sickerbecken nach. Er enthält in den obersten $50 \mathrm{~cm}$ ein Gemisch, das zu $50 \%$ aus Feinkies und Grobsand bestand, der Rest war Mittelsand und ein wenig Feinsand. Die dar-

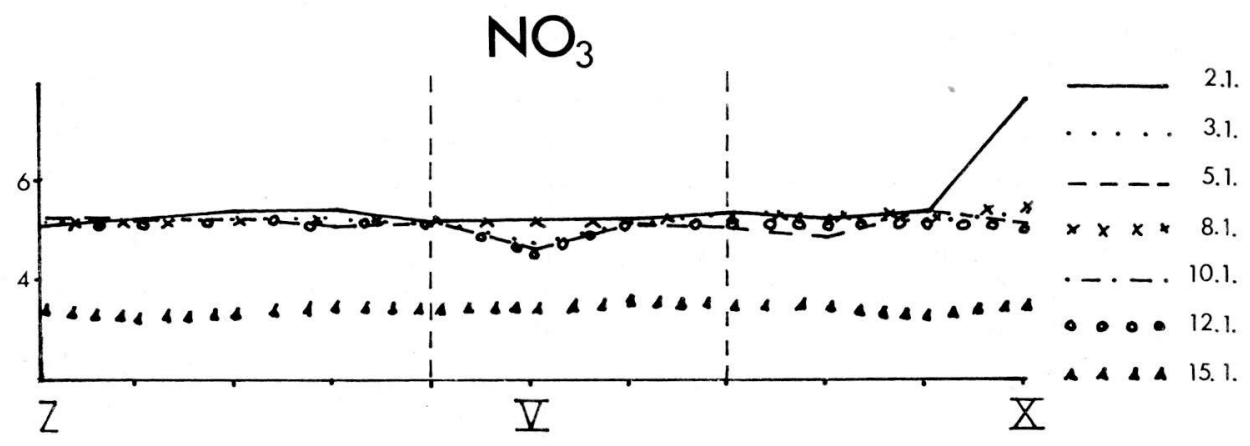

Abb. 5: Der Gehalt an $\mathrm{NO}_{3}(\mathrm{mg} / \mathrm{l})$ in den einzelnen Wasserproben zwischen Zulauf, Z, und Ablauf, X, von Filter 4 (Schichtung Grobsand-Mittelsand-Feinsand). 
unterliegende Sandpackung bestand ihrerseits zu $80 \%$ aus Mittelsand, wie er dem natürlichen Boden entspricht. (Abb. 6)

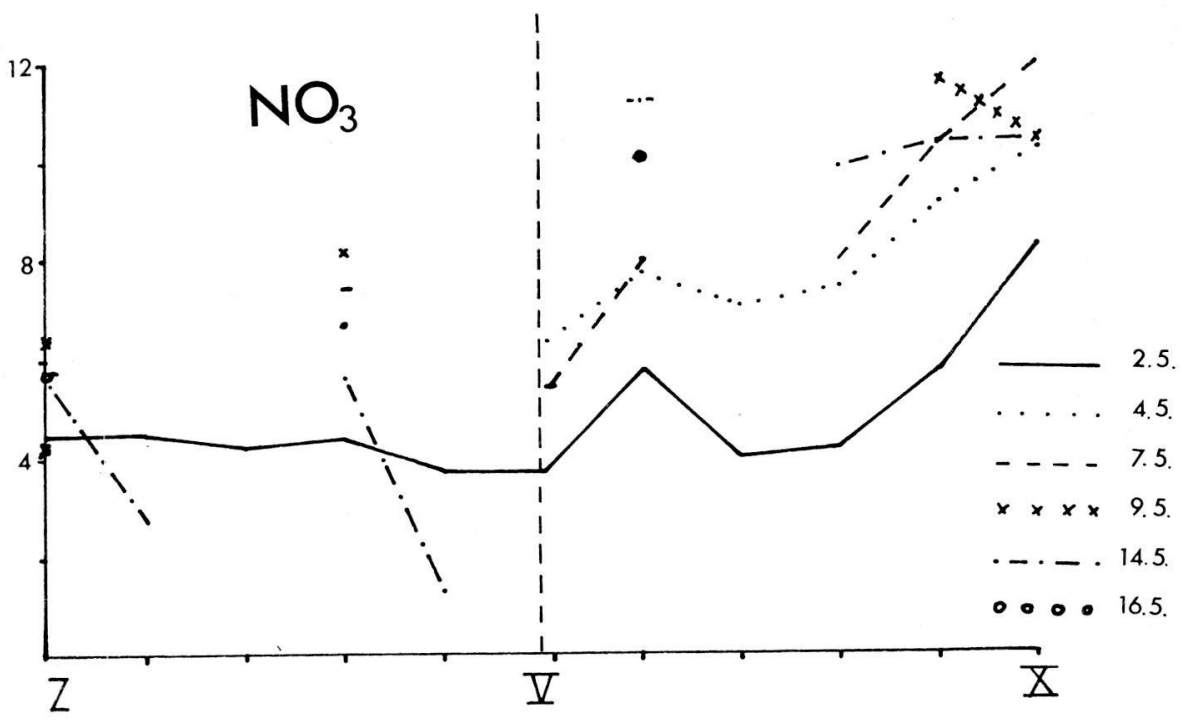

Abb. 6: Der Gehalt an $\mathrm{NO}_{3}(\mathrm{mg} / \mathrm{l})$ in den einzelnen Wasserproben zwischen Zulauf, $\mathrm{Z}$, und $\mathrm{Ab}-$ lauf, X, von Filter 5 (Schichtung Mischsand-Mittelsand).

Die $\mathrm{NO}_{3}$-Grafik läßt trotz der fehlenden Proben (die wahrscheinliche Ursache ist oben genannt) erkennen, daß die Abbaurate für eine deutliche Mineralisation spricht. Man kann also sagen, daß der Filter mit den natürlichen Bodenverhältnissen die relativ beste Siebwirkung zeigt, daß er für die interstitielle Biozönose die relativ besten Lebensbedingungen liefert.

Nachdem durch die beschriebenen technischen Maßnahmen die Abhängigkeit der Filterwirkung von der Korngröße und Zusammensetżung des Substrats nachgewiesen wurde, kam es nun darauf an, zu prüfen, ob die Wasserqualität durch biologische Maßnahmen zusätzlich verbessert werden kann.

$\mathrm{Zu}$ diesem Zweck wurde das Interstitial von Filter 5 mit Metazoen beimpft. Mit Nematoden und Rotatorien, weil sie die zahlenmäßig häufigsten waren.

Die Tiere wurden aus dem Sand der Sickerbecken gespült und nach folgendem Schema in dem vorher beschriebenen Filter 5 angereichert. (Abb. 7), Abb. 8: Eine Verlaufsbetrachtung der chemischen Analysen ist bei diesem Filter wegen der Verstopfung nicht möglich. Zur Beurteilung der Mineralisationsprozesse lassen sich nur die Anfangs- und Endwerte der einzelnen Kurven heranziehen. Diese besagen im vorliegenden Fall, daß der Wirkungsgrad des beimpften Filters kaum höher ist als derjenige der unbeimpften. 


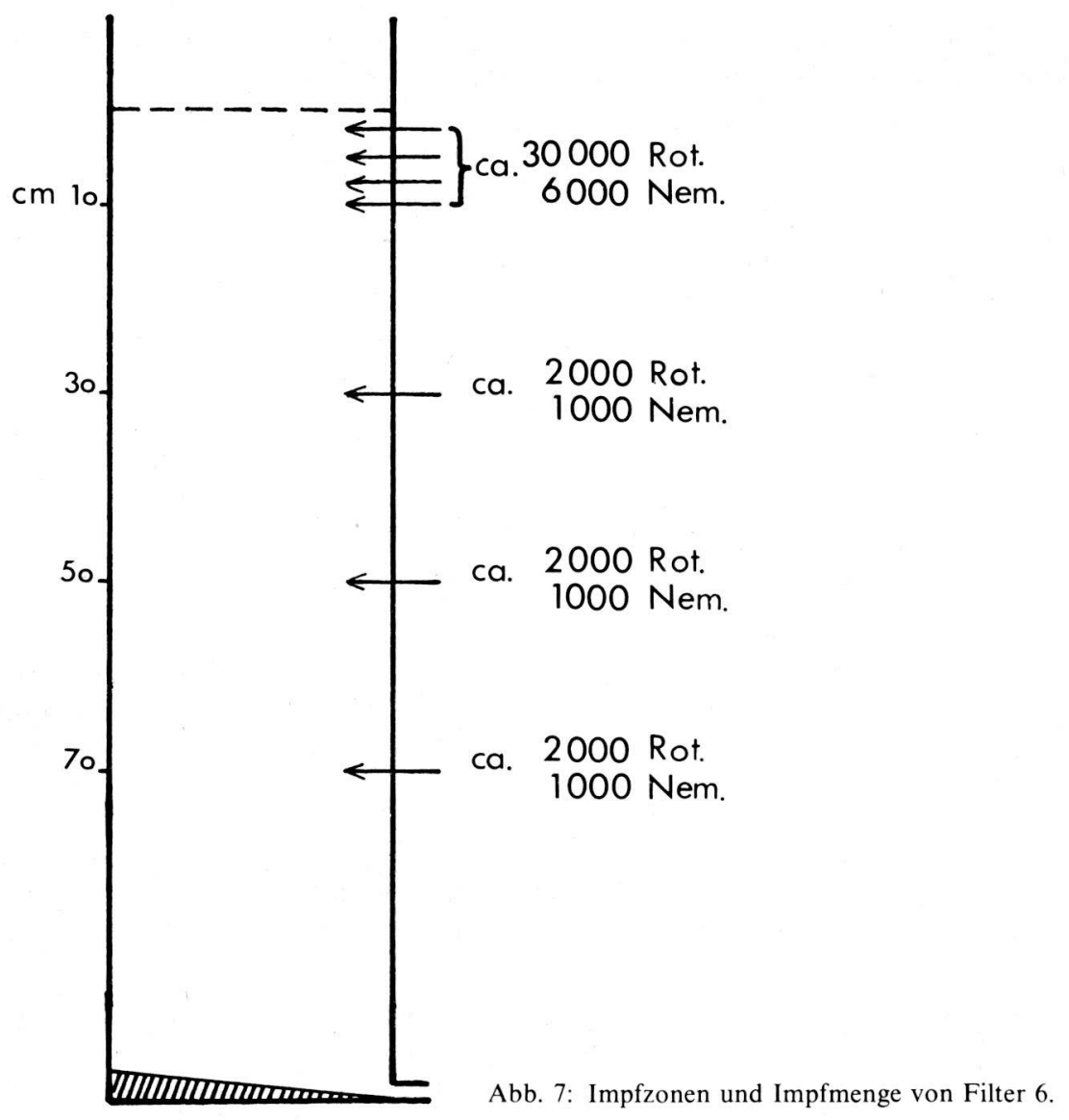

Der Wirkungsgrad $s=$ Differenz Zulauf $/$ Ablauf $(\%)$ gilt für alle gemessenen Orte und Tage, und wurde als Mittelwert für jede Substanz und jeden Filter berechnet. Auf diese Weise läßt sich die Mineralisationskraft der einzelnen Filter sehr gut miteinander vergleichen. Auch diese Methode führt zu dem Ergebnis, daß der Filter mit dem natürlichen Substrat der Sickerbecken relativ am besten arbeitet, und daß diese Wirkung durch die Beimpfung mit Metazoen nicht deutlich verbessert werden konnte.

Als Ergebnis der Untersuchungen liegt also vor, daß die Qualität des Sickerwassers durch die angewandte Methode nicht eigentlich verbessert werden konnte. Unter den beschriebenen Bedingungen scheidet das Mesopsammon, hier: Nematoden und Rotatorien, als wesentlicher Faktor für die Reinigung des Sickerwassers aus. Damit ist die eingangs gestellte Frage negativ beantwortet: diese Tiere sind in technischem Maßstab als biologische Wasserreiniger nicht einsetzbar. 


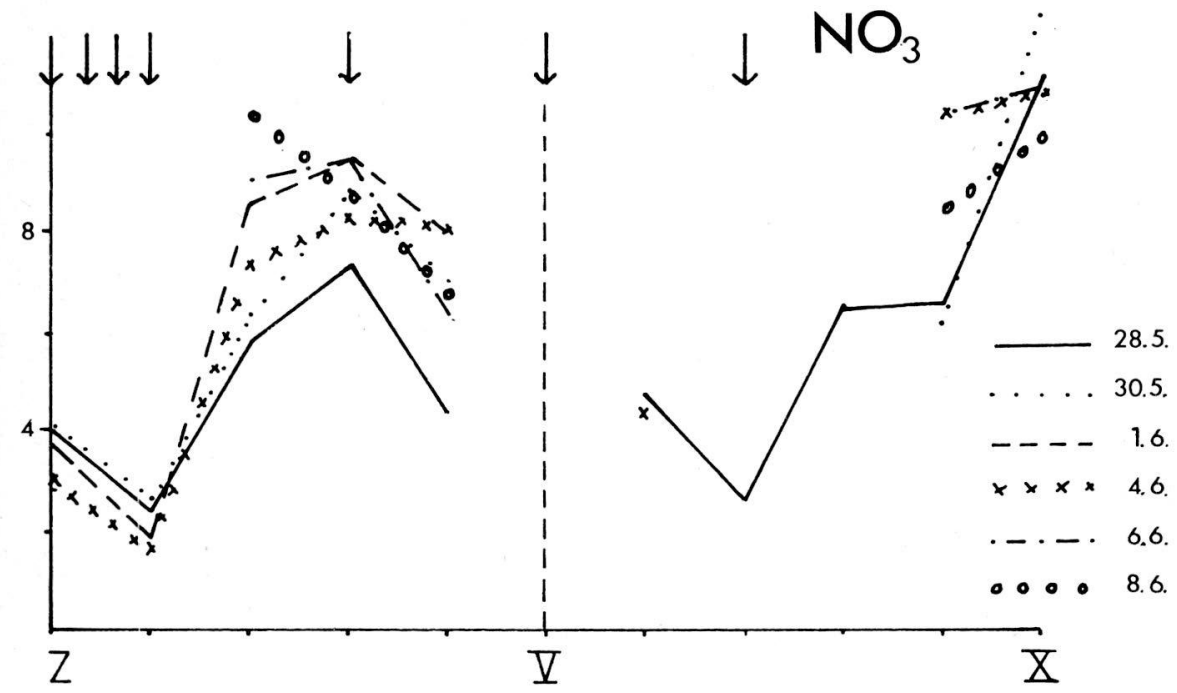

Abb. 8: Der Gehalt an $\mathrm{NO}_{3}(\mathrm{mg} / \mathrm{l})$ in den einzelnen Wasserproben zwischen Zulauf, Z, und Ablauf, X, von Filter 6.

\section{ZUSAMMENFASSUNG}

Ausgehend von der Hypothese, daß die Metazoen (Nematoden und Rotatorien) im Interstitial eines Langsamsandfilters von Bedeutung bei der Reinigung des Oberflächenwassers sind, wurde ein Filter gebaut, der die Filterkraft verschiedener Korngrößen mit den natürlichen Boden vergleicht. Es wurde festgestellt, daß die Korngrößenzusammensetzung des Sandes, der in den Sickerbecken vorliegt, die relativ beste Siebwirkung hat. Der Nachweis für die Filter erfolgte durch chemische Analysen von $\mathrm{NH}_{4} \mathrm{NO}_{3}, \mathrm{NO}_{2}, \mathrm{KMnO}_{4}$-Verbrauch und $\mathrm{O}_{2}$-Sättigung. Im vorliegenden $\mathrm{Be}$ richt sind nur die $\mathrm{NO}_{3}$ Kurven dargestellt.

Die Bedeutung der Metazoen im Verlauf der Versickerung wurde in der Weise geprüft, daß der beste Filter mit metazoischem Mesopsammon beimpft wurde.

Chemische Analysen des Wassers zeigten keine bedeutende Verbesserung der Wasserqualität gegenüber dem unbeimpften Filter.

Aufgrund dieses Ergebnisses scheint es nicht gegeben, dals die Metazoen als wichtige tierische Komponente bei der Wasserreinigung durch Sandfiltration fungieren. 